\title{
Ligadura da Veia Renal Esquerda e Preservação da Função Renal pelas Colaterais nos Tumores Retroperitoneais
}

\author{
Left Renal Vein Ligation and Preservation of Renal Function Through the Collateral Circulation after Retroperitoneal \\ Tumor Resection \\ Ligadura de la Vena Renal Izquierda y Preservación de la Función Renal por los Colaterales en los Tumores \\ Retroperitoneales
}

Rinaldo Gonçalves'; José Luiz Bravin Júnior²

\begin{abstract}
Resumo
Introduçáo: $\mathrm{O}$ retroperitônio pode apresentar diversas patologias e possui certa complexidade e mau prognóstico em virtude do íntimo contato e envolvimento de grandes vasos nas abordagens cirúrgicas. A cirurgia é a opção terapêutica associada à sobrevida prolongada. O estudo tem por objetivo demonstrar que é possível a preservação da função renal, pelas colaterais, após a ligadura ou ressecção da veia renal esquerda sem reconstrução vascular. Relato dos casos: Dois casos de pacientes com tumores retroperitoneais com invasão vascular local onde, para sua completa ressecção, fizeram-se necessárias a secção e a ligadura da veia renal esquerda. Evoluíram com preservação da função renal em razão da drenagem venosa pelas colaterais, não sendo necessária a reconstrução vascular. Além disso, foi realizada, para melhor discussão dos casos, revisão bibliográfica. Conclusão: A secção e ligadura da veia renal esquerda mostraram-se seguras nos dois pacientes tratados com ressecção de tumores retroperitoneais, não sendo a sua reconstrução obrigatória quando preservadas as colaterais para drenagem venosa.
\end{abstract}

Palavras-chave: Veias Renais; Ligadura; Neoplasias Retroperitoneais; Circulação Colateral

\begin{abstract}
Introduction: The retroperitoneum can present several pathologies that have a certain complexity and a poor prognosis due to their proximity to large vessels, the involvement of which complicates surgical approaches. Surgery is the therapeutic option associated with the longest survival. The objective of this study was to demonstrate that renal function can be preserved after ligation or resection of the left renal vein, without vascular reconstruction, if the collateral circulation remains functional. Case reports: We report two cases of patients with retroperitoneal tumors with local vascular invasion, the complete resection of which required division and ligation of the left renal vein. Postoperatively, both patients showed preserved renal function, because of collateral venous drainage, and vascular reconstruction was not necessary in either case. We also present a review of the literature on such cases. Conclusion: Division and ligation of the left renal vein proved to be safe in both of the patients undergoing retroperitoneal tumor resection, and reconstruction of the vein is not mandatory when the collateral circulation is preserved.

Key words: Renal Veins; Ligation; Retroperitoneal Neoplasms; Collateral Circulation.
\end{abstract}

Resumen

Introducción: El retroperitoneo puede presentar diversas patologías y posee cierta complejidad y mal pronóstico debido al íntimo contacto e implicación de grandes vasos en el abordaje quirúrgico. La cirugía es la opción terapéutica asociada a la supervivencia prolongada. El estudio tiene por objetivo demostrar que es posible la preservación de la función renal, por las colaterales, después de la ligadura o resección de la vena renal izquierda sin reconstrucción vascular. Relato de los casos: Dos casos de pacientes con tumores retroperitoneales con invasión vascular local donde, para su completa resección, se hizo necesaria la sección y ligadura de la vena renal izquierda. Evolucionaron con preservación de la función renal debido al drenaje venoso por las colaterales, no siendo necesaria la reconstrucción vascular. Además, se realizó, para una mejor discusión de los casos, revisión bibliográfica. Conclusión: La sección y ligadura de la vena renal izquierda se mostró segura en los dos pacientes tratados con resección de tumores retroperitoneales, no siendo su reconstrucción obligatoria cuando se preservaron las colaterales para drenaje venoso.

Palabras clave: Venas Renales; Ligadura; Neoplasias Retroperitoneales; Circulación Colateral.

\footnotetext{
${ }^{1}$ Médico-Cirurgião Oncológico. Mestre em Cirurgia-Geral pela Universidade Federal do Rio de Janeiro (UFRJ). Departamento de Cirurgia Abdominal e Pélvica do Instituto Nacional de Câncer José Alencar Gomes da Silva (INCA). Rio de Janeiro (RJ), Brasil. E-mail: rinaldo.silva@inca.gov.br.

${ }_{2}^{2}$ Médico-Residente em Cirurgia Oncológica do INCA. E-mail: bravinjr@gmail.com.

Endereço para correspondência: Rinaldo Gonçalves. Praça Cruz Vermelha, 23 - Centro. Rio de Janeiro (RJ), Brasil. CEP 20230-130.
} 


\section{INTRODUÇÃO}

O retroperitônio pode apresentar diversas patologias, incluindo uma variedade de tumores benignos e neoplasias malignas que podem ser lesôes primárias ou metastáticas. Os tumores malignos do retroperitônio ocorrem quatro vezes mais frequentemente do que lesôes benignas, representando 0,1 a $0,2 \%$ de todos os tumores malignos do corpo ${ }^{1}$.

Em razão da complexidade cirúrgica e do mau prognóstico a longo prazo, o envolvimento de grandes vasos tem sido tradicionalmente considerado um fator limitante para ressecção de tumores retroperitoneais. Contudo, os avanços tanto nas técnicas cirúrgicas como nos cuidados peroperatórios tornaram as ressecçôes vasculares importantes opçóes terapêuticas para esses pacientes. Atualmente, a ressecçáo radical em bloco do segmento venoso afetado permanece como o único tratamento associado à sobrevida prolongada ${ }^{2-3}$.

Particularmente, em relação aos sarcomas retroperitoneais, a veia cava em sua porção perirrenal representa o segmento vascular mais envolvido ${ }^{4-6}$, seja por invasão direta ou por origem nessa localização, sendo necessária sua ressecção para obtenção de margens livres. Pela característica anatômica da presença de colaterais ${ }^{7-8}$, não há consenso na literatura sobre a necessidade de reconstrução da veia renal esquerda após a ressecção da veia cava justarrenal. Alguns autores preconizam a sua reconstrução com o objetivo de prevenir congestáo venosa do rim e consequente deterioração da função renal ${ }^{9-10}$, enquanto outros favorecem a sua ligadura direta sem reconstrução pela praticidade, menor tempo cirúrgico e menor risco de sangramento envolvidos ${ }^{11-12}$.

Temos por objetivo demonstrar que, após estudo radiológico dos casos e confirmação de circulação colateral, é possível a preservação da função renal esquerda após a ligadura ou ressecção da veia renal esquerda sem reconstrução vascular, sendo relatados dois casos de tumores retroperitoneais em que, para sua completa ressecção, fizeram-se necessárias a secção e a ligadura da veia renal esquerda. Além disso, para maior discussão sobre o tema, foi realizada revisão bibliográfica.

O trabalho proposto é um estudo observacional descritivo, sendo realizada a seleçáo dos pacientes de acordo com coleta dos dados clínicos e radiológicos, pela busca direta ao prontuário, realizada no Instituto Nacional de Câncer José Alencar Gomes da Silva (INCA) com aprovação pelo Comitê de Ética em Pesquisa (CEP) da instituição, sob o número CAAE: 79253717.6.0000.5274, e isenção do Termo de Consentimento Livre e Esclarecido (TCLE).

\section{RELATO DOS CASOS}

Primeiro caso: Paciente, sexo feminino, 56 anos, com quadro de dor em barra em abdômen superior e dorso, com um ano de duração dos sintomas. Apresentando na ressonância magnética (RNM) formação retroperitoneal indissociável do processo uncinado do pâncreas e da veia cava inferior (Figura 1), em sua porção infrarrenal, com extensão ao óstio da veia renal esquerda, poupando a inserção das veias gonadal e suprarrenal deste lado.

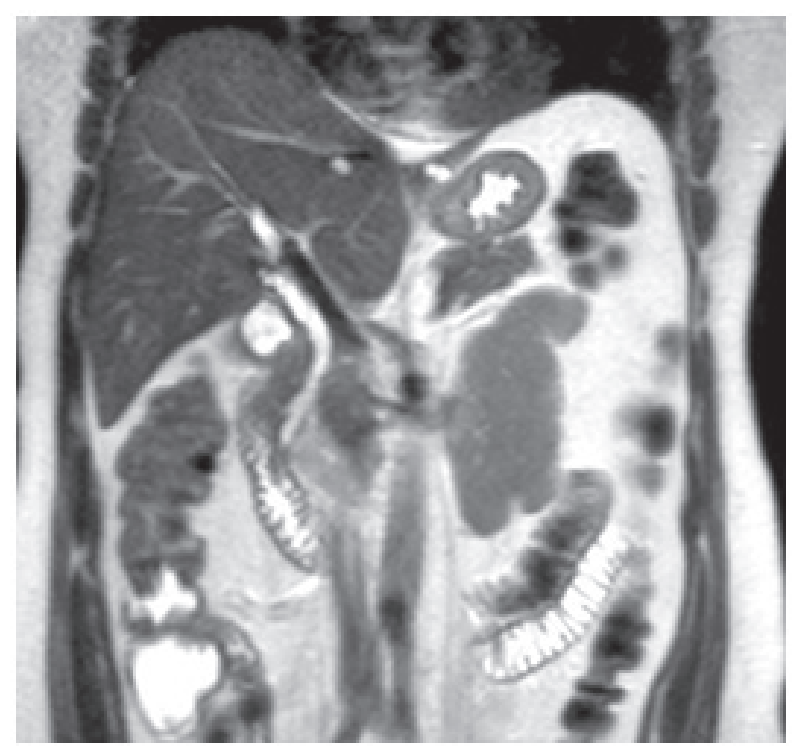

Figura 1. Exame de imagem com evidência de lesão tumoral, retroperitoneal, comprometendo veia cava inferior

A ultrassonografia (USG) endoscópica confirmou os achados, sendo realizada biópsia por punção ecoguiada com laudo histológico e imuno-histoquímico de leiomiossarcoma.

$\mathrm{Na}$ apresentação cirúrgica, foram confirmados os achados imaginológicos pré-operatórios, com a presença lesão sarcomatosa originada em veia cava inferior em sua porção infrarrenal, e extensão até o óstio da veia renal esquerda. $O$ tratamento cirúrgico consistiu na excisão cirúrgica da lesão retroperitoneal, juntamente com segmento de veia cava correspondente e secção e ligadura da veia renal esquerda em sua inserção. Optou-se por reconstrução da veia cava inferior com interposição de prótese de Dacron. Após confirmação da presença das veias gonadal e suprarrenal como tributárias da veia renal esquerda, decidiu-se pela sua náo reconstrução, sendo observada boa drenagem venosa com consequente aspecto não congestivo do rim esquerdo ao término do procedimento.

Paciente teve evolução satisfatória, recebendo alta hospitalar sem intercorrências no pós-operatório. No 
seguimento ambulatorial, foram realizados exames complementares para avaliação da função renal, apresentando exame de urina com ausência de proteinúria. A angiotomografia computadorizada (angio-TC) evidenciou drenagem da veia renal esquerda por meio das veias gonadais e suprarrenais esquerdas, nefrograma com aporte sanguíneo renal bilateral preservado, fase parenquimatosa e excretora em tempo hábil, e cintilografia renal estática com DMSA 99mTc evidenciando função renal diferencial relativa de $44 \%$ no rim direito e $56 \%$ no rim esquerdo (Figura 2).

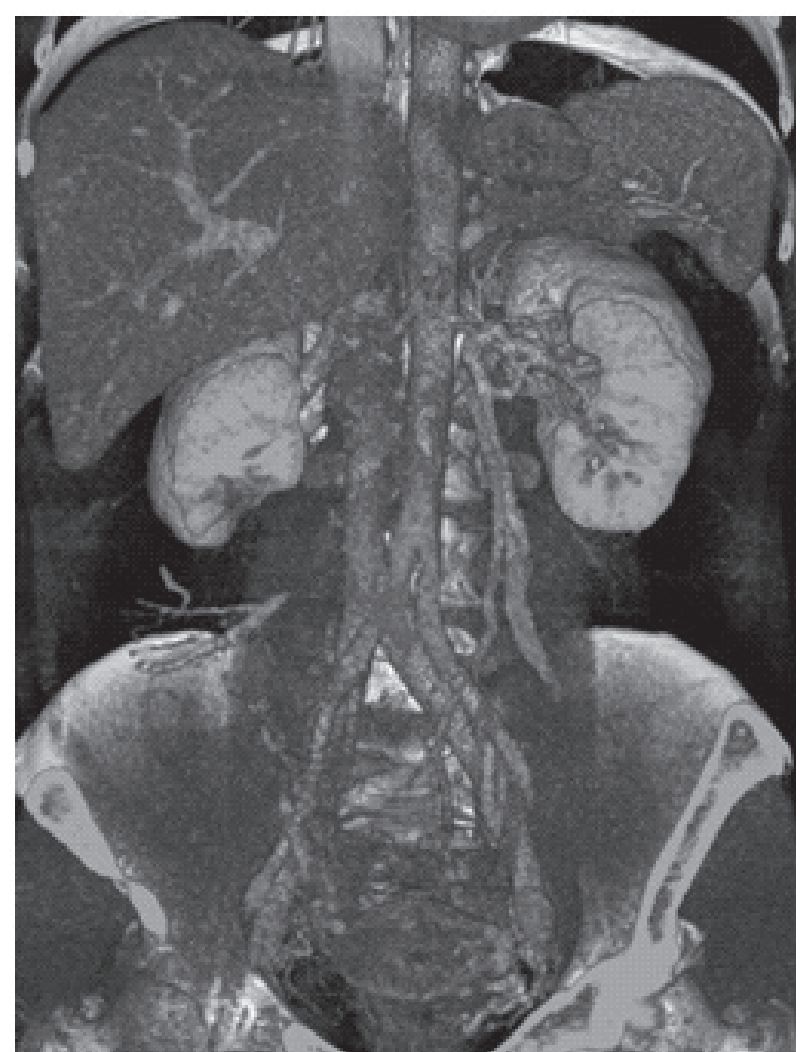

Figura 2. Angio-TC pós-operatória evidenciando drenagem da veia renal pelas colaterais, veias gonadais e suprarrenais esquerdas

Segundo caso: Paciente, sexo feminino, 37 anos, apresentando quadro clínico de dor abdominal em flanco direito, com duas semanas de evolução, associado à disúria. Nega etilismo, tabagismo, comorbidades ou cirurgias prévias. Sendo tratada inicialmente com antibioticoterapia e conduzida investigação com USG de vias urinárias onde foi identificada massa em topografia suprarrenal direita.

Optou-se pela realização de tomografia computadorizada (TC) do abdômen e pelve para melhor investigação, na qual foi evidenciada massa, medindo 12,8 x 11,4 x $6,9 \mathrm{~cm}$, em íntimo contato com adrenal direita e deslocando mediamente a artéria renal direita e a veia cava inferior, com envolvimento parcial desta (Figura 3).

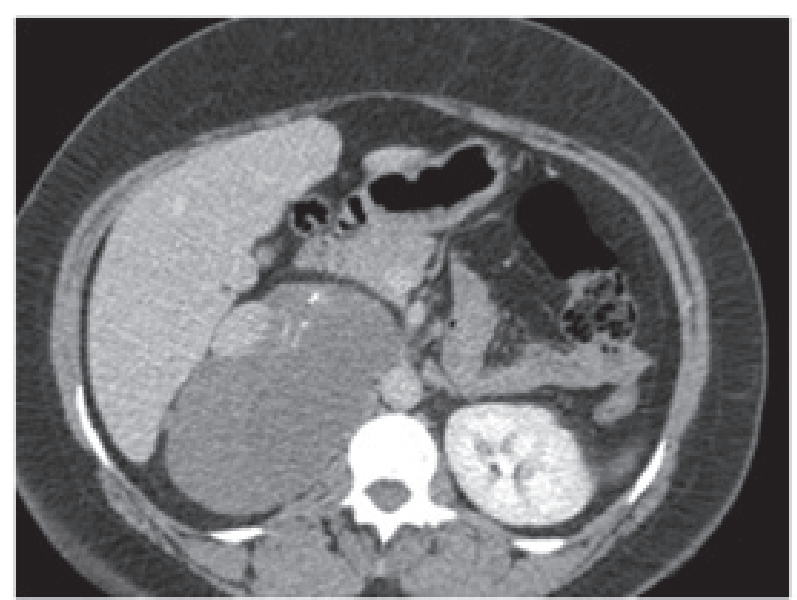

Figura 3. Lesão tumoral, medindo 12,8 x 11,4 ×6,9cm, em íntimo contato com adrenal direita e deslocando mediamente artéria renal direita e veia cava inferior

A abordagem escolhida foi a cirúrgica, apresentando, no achado intraoperatório, lesão tumoral retroperitoneal volumosa, em topografia de glândula adrenal direita, fortemente aderida ao duodeno, hilo hepático, tronco celíaco e vasos mesentéricos. A lesão se estendia posteriormente à veia cava e veia renal esquerda, determinando deslocamento medial e anterior destas últimas. Durante a ressecção cirúrgica, fizeram-se necessárias a secção e a ligadura da veia renal esquerda para possibilitar a mobilização e liberação da massa tumoral dos planos profundos. Uma vez constatada a presença da veia gonadal e suprarrenal como colaterais da veia renal esquerda, optou-se pela não reconstrução vascular. $\mathrm{O}$ laudo histopatológico revelou feocromocitoma.

Paciente teve evolução satisfatória, recebendo alta hospitalar sem intercorrências no quinto dia de pós-operatório. No seguimento ambulatorial, foram realizados exames complementares para avaliação da função renal. Foi apresentado exame de urina dentro da normalidade e ausência de proteinúria; e a angio-TC e cintilografia renal evidenciaram boa perfusão renal bilateral (Figura 4).

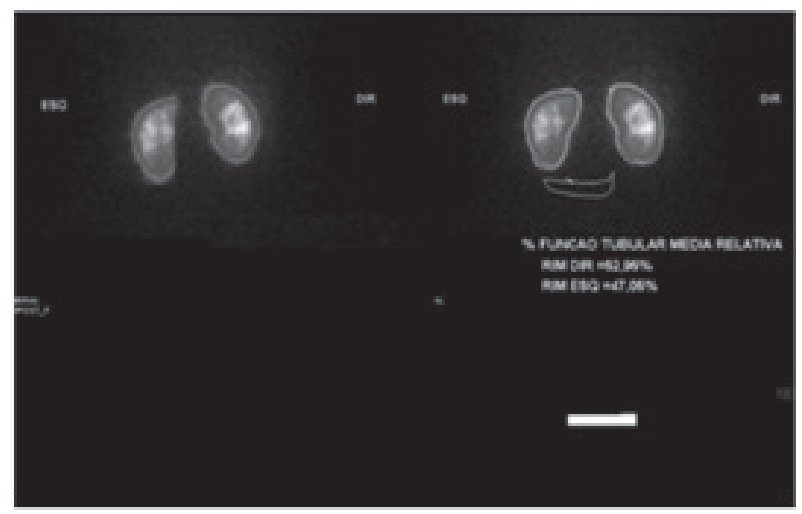

Figura 4. Cintilografia renal evidenciando boa perfusão renal bilateral 


\section{DISCUSSÃO}

O retroperitônio representa um espaço anatômico cirurgicamente desafiador, com múltiplas estruturas vitais dentro dos seus limites, tornando as ressecçôes de tumores localizados nessa topografia procedimentos tecnicamente complexos. Tumores dessa região se apresentam habitualmente em estágios avançados, muitas das vezes, em íntimo contato ou com invasão de órgãos e estruturas adjacentes, necessitando, por vezes, de ressecçôes vasculares para a obtenção de margens oncologicamente adequadas. Especialmente no que diz respeito aos sarcomas retroperitoneais, a veia cava corresponde à estrutura vascular mais envolvida ${ }^{13-17}$, seja por invasáo direta ou se originando nesse vaso, sendo a região perirrenal a mais habitualmente acometida $a^{4-6}$, o que implica não raramente na ressecção associada das veias renais. Similarmente ao que ocorre em relação à veia cava infrarrenal, na qual não há consenso sobre a necessidade de reconstrucáo ${ }^{18}$, no que diz respeito à veia renal esquerda, alguns autores advogam a sua ligadura direta, enquanto outros preconizam a reconstrução de maneira rotineira.

Um estudo avaliando 47 pacientes submetidos à ressecção de veia cava inferior para excisão tumoral obteve 19 reimplantes de veias renais, tanto direita quanto esquerda ${ }^{18-19}$. Já outro estudo envolvendo 41 pacientes submetidos a ressecçóes cavais para tratamentos tumorais relatou oito ligaduras de veia renais esquerda ${ }^{20}$.

No primeiro caso descrito, o tumor era originado da veia cava no seu segmento perirrenal. Foi realizada a ressecção do segmento da veia cava envolvido juntamente com o óstio da veia renal esquerda. Pela ausência de colaterais, foi optado pela reconstrução da cava. Confirmada a presença de colaterais, para as veias gonadal e suprarrenal, optou-se pela ligadura e não reconstrução da veia renal esquerda.

O segundo caso descrito envolveu uma paciente com massa retroperitoneal retrocaval em topografia de inserção das renais, determinando deslocamento anatômico anterior importante desses vasos. Durante a dissecção cirúrgica, foi constatada dificuldade significativa de mobilização da massa tumoral em razão do íntimo contato desta com a face posterior das veias cava e renal esquerdas. Optou-se por secção e ligadura da veia esquerda justacaval como estratégia para a obtenção de exposição cirúrgica adequada, permitindo a completa mobilização e liberação da tumoração da parede abdominal posteriormente, e das veias cava e renal esquerdas anteriormente.

A secção da veia renal esquerda tem sido empregada por cirurgiōes vasculares como estratégia para a melhor exposição da aorta justarrenal quando no tratamento cirúrgico de aneurismas ou doença oclusiva aórtica ${ }^{21-23}$.
Embora esse procedimento seja pouco descrito para melhor acesso e exposição no contexto do tratamento de tumores retroperitoneais, essa estratégia mostrou-se válida e segura no paciente do estudo, permitindo uma abordagem cirúrgica com melhor exposiçáo e menor risco de sangramento, de maneira similar ao obtido quando essa estratégia é utilizada na abordagem das patologias da aorta justarrenal. Estudo publicado em 1997 no Sao Paulo Medical Journal avaliou um total de 342 nefrectomias em doadores vivos para transplantes, sendo 331 à esquerda e 31 à direita. Foi constatado que a veia renal esquerda tinha duas tributarias (veia suprarrenal e gonadal) em $100 \%$ dos casos e uma ou mais veias renolombares em $65 \%$ dos casos. A presença dessas tributárias faz com que a ligadura da veia renal sem reconstrução seja possível, fazendo-se a drenagem venosa por meio delas. Entretanto, alguns relatos sugerem que a não reconstruçáo da veia renal esquerda poderia levar à deterioração da função renal, com elevação da creatinina e diminuição da taxa de filtração sendo observadas em algumas séries. Essas alteraçóes não foram notadas de forma uniforme em todas as séries envolvendo tratamento de aneurismas abdominais. Em estudo envolvendo 56 casos de secção e ligadura da veia renal esquerda no tratamento de patologias aórticas, o nível de creatinina pré-operatório foi de $1,1 \mathrm{mg} / \mathrm{dL}(0,7-$ $2,4 \mathrm{mg} / \mathrm{dL}$ ), similar ao encontrado no momento da alta hospitalar $(1,1 \mathrm{mg} / \mathrm{dL}$, variação $0,6-2,1 \mathrm{mg} / \mathrm{dL})$. Ainda nessa série, foi observado que, no seguimento de longo tempo, somente dois pacientes apresentaram alteraçôes na função renal, creditadas à nefroesclerose hipertensiva e não à ligadura da veia renal em $\mathrm{si}^{24}$. Em uma pesquisa avaliando 261 pacientes com aneurismas de aorta submetidos a tratamento cirúrgico, 45 pacientes que tiveram secçáo e ligadura da veia renal esquerda foram comparados com 212 pacientes que náo tiveram esse procedimento realizado. Os níveis de creatinina foram semelhantes nos dois grupos, nos primeiro e sétimo dias pós-operatórios, assim como nas semanas. Esses dados sugerem que a ligadura da veia renal esquerda é segura do ponto de vista da função renal, com alteraçóes observadas de pequena repercussão clínica ou mesmo ausentes ${ }^{25}$.

Diferente do observado nas séries envolvendo tratamento de aneurisma de aorta abdominal justarrenal, em que existe a necessidade de clampeamento arterial, com consequente período de isquemia renal e mesmo reimplante de artéria renal em alguns casos, nos casos apresentados, não foi realizada qualquer intervenção arterial, não determinando, portanto, qualquer período de isquemia renal. Esse fato sugere maior segurança relativa à funçáo renal na utilização desse procedimento para tratamento de tumores retroperitoneais, em que o clampeamento aórtico e da artéria renal não seja empregado. De fato, em 
estudo envolvendo 41 pacientes submetidos à ressecção de tumores retroperitoneais associados à ressecção de veia cava, não foi observada qualquer alteração da função renal nos oito pacientes, cujas ligadura e secção da veia renal esquerda foram associadas à nefrectomia direita, reforçando a segurança daquele procedimento ${ }^{20}$.

\section{CONCLUSÃO}

Em conclusão, a secção e a ligadura da veia renal esquerda mostraram-se seguras nos dois pacientes tratados com ressecção de tumores retroperitoneais, não sendo a sua reconstrução obrigatória quando preservadas as colaterais para drenagem venosa.

\section{DECLARAÇÃO DE CONFLITO DE INTERESSES}

Nada a Declarar.

\section{REFERÊNCIAS}

1. Osman S, Lehnert BE. A comprehensive review of the retroperitoneal anatomy, neoplasms, and pattern of disease spread. Current probl diagn radiol. 2013;42(5):191-208.

2. Kieffer E, Alaoui M, Piette JC, Cacoub P, Chiche L. Leiomyosarcoma of the inferior vena cava: Experience in 22 cases. Ann Surg. 2006;244(2):289-95.

3. Hardwigsen J, Baqué P, Crespy B, Moutardier V, Delpero JR, Le Treut YP. Resection of the inferior vena cava for neoplasms with or without prosthetic replacement: A 14-patients series. Ann Surg. 2001;233(2):242-9.

4. Mingoli A, Cavallaro A, Sapienza P, Di Marzo L, Feldhaus RJ, Cavallari N. International registry of inferior vena cava leiomyosarcoma: Analysis of a world series on 218 patients. Anticancer Res. 1996;16(5B):3201-5.

5. Kieffer E, Alaoui M, Piette JC, Cacoub P, Chiche L. Leiomyo-sarcoma of the inferior vena cava: experience in 22 cases. Ann Surg. 2006;244(2):289-95.

6. Ramponi F, Kench J, Simring D, Crawford M, Abadir E, Harris JP. Early diagnosis of an asymptomatic leiomyosarcoma of the inferior vena cava prior to caval obstruction. J Vasc Surg. 2012;55(2):525-8.

7. Anson BJ, Cauldwell EW, Pick JW, Beaton LE. The anatomy of the pararenal system of veins, with comments on the renal arteries. J Urol. 1948;60(5):714-37.

8. Baptista-Silva JCC, Cal RGR, Castro MJ, Veríssimo MJM, Câmara ALG. Estudo anatômico das veias renais e suas variações, observação durante as nefrectomias em doadores- vivos. Rev Col Bras Cir. 1995;22(2):18.

9. West CA, Noel AA, Bower TC, Cherry Junior KJ, Gloviczki P, Sullivan TM, et al. Factors affecting outcomes of open surgical repair of pararenal aortic aneurysms: a 10-year experience. J Vasc Surg. 2006;43(5):921-7; discussion 927-8.

10. AbuRahma AF, Robinson PA, Boland JP, Lucente FC. The risk of ligation of the left renal vein in resection of the abdominal aortic aneurysm. Surg Gynecol Obstet. 1991;173(1):33-6.

11. James EC, Fedde CW, Khuri NT, Gillespie JT. Division of the left renal vein: a safe surgical adjunct. Surgery 1978;83(2):151-4.

12. Adar R, Rabbi I, Bass A, Papa MZ, Schneiderman J, Walden R. Left renal vein division in abdominal aortic aneurysm operations. Effect on renal function. Arch Surg 1985;120(9):1033-6.

13. National Institute for Health and Clinical Excellence. Improving Outcomes for People with Sarcoma: The Manual. London: niCE; 2006.

14. Strauss DC, Hayes AJ, Thway K, Moskovic EC, Fisher C, Thomas JM. Surgical management of primary retroperitoneal sarcoma. Br J Surg. 2010; 97(5):698-706.

15. Clark ma, Fisher C, Judson i, Thomas Jm. Soft-tissue sarcomas in adults. N Engl J Med. 2005;353(7):701-11.

16. Burke AP, Virmani R. Sarcomas of the great vessels. A clinicopathologic study. Cancer. 1993;71(5):1761-73.

17. Blansfield JA, Chung H, Sullivan TR Jr et al. Leiomyosarcoma of the major peripheral arteries: Case report and review of the literature. Ann Vasc Surg 2003;17(5):565-70.

18. Mingoli A, Feldhaus RJ, Cavallaro A, Stipa S. Leiomyosarcoma of the inferior vena cava: Analysis and search of world literature on 141 patients and re- port of three new cases. J Vasc Surg. 1991;14(5):688-99.

19. Kuehnl A, Schmidt M, Hornung HM, Graser A, Jauch KW, Kopp R, et al. Resection of malignant tumors invading the vena cava: perioperative complications and long-term follow-up. J Vasc Surg. 2007;46(3):533-40.

20. Goto H, Hashimoto M, Akamatsu D, Shimizu T, Miyama N, Tsuchida K, et al. Surgical Resection and Inferior Vena Cava Reconstruction for Treatment of the Malignant Tumor: Technical Success and Outcomes. Ann Vasc Dis. 2014;7(2):120-6.

21. RoysterTS, Lacey L, Marks RA. Abdominal aortic surgery and left renal vein. Am J Surg. 1974;127(5):552-4.

22. McCombs PR, DeLaurentis DA. Division of the left renal vein. Guidelines and consequences. Am J Surg. 1979;138(2):257-63.

23. Neal HS, Shearburn EW. Division of the left renal vein as an adjunct to resection of abdominal aortic aneurysm. Am J Surg. 1967;113(6):763-5.

24. Samson RH, Lepore MR Jr, Showalter DP, Nair DG, Lanoue JB. Long-term safety of left renal vein division and ligation to expedite complex abdominal aortic surgery. J Vasc Surg. 2009;50(3):500-4. 
Gonçalves R, Bravin Júnior JL

25. Mehta T, Wade RG, Clarke JM. Is it safe to ligate the left renal vein during open abdominal aortic aneurysm repair? Anna Vasc Surg. 2010;24(6):758-61.

Recebido em 15/2/2018

Aprovado em 30/5/2018 